No. 1

Enero-Diciembre 2016 ISSN 1794-8231

\title{
Proyecto de vida de los adolescentes del área metropolitana de Cúcuta (zona rural escolar corregimiento de san Faustino)
}

\author{
Life-project of adolescents in the metropolitan area of cúcuta \\ (san faustino rural school area)
}

\section{Projeto de vida dos adolescentes da zona rural escolar da área metropolitana de cúcuta (comuna noroeste, corregimiento de san faustino)}

\author{
Edgar Alfonso Santos-Contreras ${ }^{1}$, Dania Paola Ortega-Pérez ${ }^{2}$
}

Forma de citar: E. A. Santos-Contreras, D. P. Ortega-Pérez, "Proyecto de vida de los adolescentes del área metropolitana de Cúcuta (zona rural escolar corregimiento de san Faustino)”, Eco.Mat. vol. 7, no. 1, pp. 112-120, 2016

Recibido:

Mayo 25 de 2015

Aceptado:

Agosto 31 de 2015

\section{2}

\section{Resumen}

El presente trabajo se inscribe dentro del estudio de las condiciones humanas en sus múltiples dimensiones que permiten el desarrollo personal y profesional, siendo el proyecto de vida una herramienta que busca orientar nuestro crecimiento personal, por medio de la identificación de aquellas metas que deseamos lograr y de las capacidades que poseemos, se percibe en las instituciones educativas y en otros contextos socioculturales en el área metropolitana de Cúcuta, un alto porcentaje de adolescentes con una carencia en la proyección de sí mismos, causados probablemente por la falta de su autoconocimiento, la influencia directa e indirecta de factores familiares, sociales, económicos y culturales de la región que durante tanto tiempo se ha mantenido geográficamente aislada y estancada del contexto nacional e internacional, por su misma condición de frontera. Para el desarrollo de este objetivo nos basamos fundamentalmente en la teoría de la jerarquía de necesidades humanas [1], la psicología humanista [2] y los proyectos de vida y planteamiento estratégico personal [3], entre otras. La metodología empleada se enmarca en la denominada investigación descriptiva que conlleva a fundamentalmente en caracterizar un fenómeno o situación concreta indicando sus rasgos más peculiares o diferenciadores. Del mismo modo se muestran los resultados obtenidos y se concluye que todos los adolescentes buscan su autorrealización, pues desde su etapa escolar van reconociendo la importancia de trazar metas y enmarcarlas en un plan aunque no sea escrito y estructurado, al tiempo que identifican factores que influyen en la construcción del mismo.

Palabras clave: adolescente, escolar, proyecto, vida.

\begin{abstract}
This work is part of the study of the human condition in its many dimensions that allow personal and professional development, with life-project being a tool which seeks to guide our personal growth, through the identification of those goals that we want to achieve and the capabilities we own. In educational institutions and other sociocultural contexts in the metropolitan area of Cucuta, a high percentage of adolescents with a gap in the projection of themselves is perceived, likely caused by the
\end{abstract}


lack of self-knowledge, the direct and indirect influence of family, social, economic and cultural factors from the region which for so long has remained geographically isolated from national and international context, due to its border condition. To develop this goal we rely primarily on the theory of hierarchy of human needs [1] , humanistic psychology [2] and life projects and strategic planning staff [3] among others. The methodology is part of the so-called descriptive research based on characterization of phenomena or situations indicating the most peculiar or differentiating facts. Results are shown and it is concluded that all adolescents look for self-realization, as since their school stage they start recognizing the importance of setting goals and set them in a plan even if it is not written or structured, while identifying factors influencing in construction of that plan.

Keywords: adolescent, life, project, schoolar.

\section{Resumo}

O presente trabalho é parte do estudo das condições humanas em suas múltiplas dimensões que permitem o desenvolvimento pessoal e professional, sendo o projeto de vida uma ferramenta que procura orientar nosso crescimento pessoal, por médio da identificação de aquelas metas que desejamos atingir e das capacidades que temos. Percebe-se nas instituições educativas e em outros contextos socioculturais na área metropolitana da cidade de Cúcuta, uma alta porcentagem de adolescentes com uma carência na projeção de si mesmos, causados provavelmente pela falta de seu autoconhecimento, a influência direta e indireta de fatores familiares, sociais, económicos e culturais da região que por tanto tempo tem-se mantido geograficamente isolada e estagnado do contexto nacional e internacional, por sua mesma condição de fronteira. Para o desenvolvimento deste objetivo nos baseamos fundamentalmente na teoria da jerarquia de necessidades humanas [1], a psicologia humanista [2] e os projetos de vida e planejamento estratégico pessoal [3] entre outras. A metodologia empregada faz parte da denominada pesquisa descritiva que leva fundamentalmente a caracterizar um fenómeno ou situação concreta indicando suas características mais peculiares ou diferenciadoras. Do mesmo modo, se mostram os resultados obtidos e se conclui que todos os adolescentes procuram sua autorrealização, porque a partir de sua etapa escolar vão reconhecendo a importância de traçar metas e emoldurá-las num projeto de vida, embora não seja escrito e estruturado, ao tempo que identificam fatores que influem na construção do mesmo.

Palavras-chave: adolescente, escolar, projeto, vida

\section{Introducción}

En las diversas experiencias y relaciones con otras personas, ya sea en la familia como en otros círculos sociales, se pueden conocer distintas formas de vivir y enfrentar la vida: hay quienes viven el «cada día» esperando sobrevivir al día siguiente; hay quienes viven esta situación, pero a la vez realizan planes; hay quienes planifican lo que desean hacer en corto, mediano o largos plazos y en función de ello organizan su diario vivir.
Los seres humanos siempre van en busca de conseguir una serie de fines o metas en la vida. Algunos de esos fines son insignificantes y se persiguen inconscientemente, pero otros sirven de guía para la vida. La pregunta clave es: ¿Se dedica un tiempo pausado a plantear por qué y para qué se hacen las cosas? Dicho de otro modo: ¿nuestra vida se mueve a base de impulsos sin sentido, o nos paramos a pensar lo que queremos llegar a ser y como conseguirlo? La mayoría de las personas ni 
siquiera han fijado un objetivo claro en su vida [4]

La necesidad de todo ser humano de alcanzar su desarrollo pleno y lograr sus metas, es fundamental a lo largo de la vida para mirar con satisfacción y con optimismo lo que el entorno le presenta, aún se trate de limitaciones o amenazas, pues estas significarán antes un reto que una sensación de derrota o de obstáculo definitivo. Esta es la dimensión singular de la vida del ser humano, porque puede transformar su realidad, puede proyectar y construir, tomando decisiones y actuando en un margen de libertad, enmarcado desde la concepción que cada uno de los adolescentes han asumida en relación al proyecto de vida, hasta la influencia de la institución educativa en la cual se encuentran, pasando por los factores internos y externos que implican dicha proyección, y las tendencias profesionales u ocupacionales que priorizan los estudiantes. Se comprende entonces el proyecto de vida, como un sistema principal de la persona en su dimensionalidad esencial de vida, un modelo ideal-real complejo de la dirección perspectiva de su vida [5].

Cuando se habla de proyecto para los adolescentes se plasman todos aquellos planes que ellos deseen cumplir, tomando en cuenta los diferentes ámbitos de la vida; implica un acto de consciencia, valentía, entrega, madurez y de toma de decisiones, para dar sentido a la existencia entendida como rumbo (a dónde quiero llegar) y significado (qué representa para mí).

Entonces, en la interacción diaria de los adolescentes, hay sueños, ilusiones y anhelos de cada día ser mejores personas, vivir mejor, con mayores comodidades y poder ser alguien [6], pero entre este ideal y la realidad de su presente hay un camino por recorrer, el cual será más asequible en la medida que conozcan y elaboren su proyecto de vida. La importancia de la realización del proyecto de vida no sólo radica en la identificación de aquello que se quiere lograr, sino que a través del establecimiento de metas permite identificar o generar los recursos o medios para su cumplimiento [7].

El proyecto de vida debe ser entendido como una formación psicológica que cobra especial importancia en la autorrealización del individuo, donde se estructuran los objetivos fundamentales de la vida [8]. Se constituye en un elemento determinante en la vida del sujeto e indicador esencial del desarrollo de la personalidad, constituyendo, además, una importante expresión de la motivación humana superior.

Actualmente, encontramos un gran número de adolescentes que pasan parte de su vida, experimentando una gran variedad de circunstancias, queriendo probar en la mayoría de los casos la suerte que el destino les tenga preparado.

Muchos adolescentes que al contar con las comodidades y oportunidades, logran concretar un proyecto de vida, realizando primero una capacitación académica en un campo profesional específico. Otros, en cambio, como se exponía en un principio, inician una aventura de exploración donde sólo a través de lo empírico, van acoplándose a lo que el medio les permita hacer. Y en casos aún más desfavorables, hay adolescentes que teniendo las condiciones económicas, sociales, y demás a su favor, también se limitan sólo a experimentar en diferentes ramas de acción, sin lograr concretar una meta fija que le permita un desarrollo óptimo de sí mismo.

En el trabajo realizado de orientación y proyecto de vida para estudiantes de educación secundaria [9] identificaron factores internos y externos que inciden en la estructuración del proyecto de vida de los estudiantes del colegio. Determinaron el grado de escolaridad en el que el estudiante tiene establecido su proyecto 
de vida y permite diseñar estrategias de orientación para la estructuración del proyecto de vida de los estudiantes, pues de la misma manera se logró determinar que además de los factores intrínsecos y extrínsecos también hay factores experienciales del contexto como las expectativas personales y tendencias profesionales y ocupacionales que motiva al adolescente a determinar su proyecto de vida desde temprana edad.

\section{Cuerpo del artículo}

Desde el planteamiento de la investigación, se elige un tipo de investigación descriptiva la cual consiste, fundamentalmente en caracterizar un fenómeno o situación concreta indicando sus rasgos más peculiares o diferenciadores [10].

Se observó el fenómeno de los proyectos de vida en los adolescentes del sector rural del municipio de San José de Cúcuta, es decir, en un contexto natural, pertenecientes al sector educativo del corregimiento, que luego fueron analizados en toda la data obtenida de la muestra objeto de estudio.

La población se precisa como un conjunto de elementos que presentan características comunes. En este caso de estudio la población o universo está representada por los estudiantes de la Institución Educativa Colegio Fray Manuel Álvarez, del Corregimiento de San Faustino, zona rural del municipio San José de Cúcuta que se encuentran entre los 14 y 21 años de edad, de donde se seleccionó una muestra utilizando la fórmula de probabilidades para poblaciones definidas, conociendo el tamaño de la misma, como se muestran a continuación las tablas I y II respectivamente.

Tabla II. Estudiantes por edad de la Institución Educativa Fray Manuel Álvarez, corregimiento de San Faustino, zona rural del municipio de San José de Cúcuta

Tabla I Edad Estudiantes

\begin{tabular}{|c|c|c|c|c|c|c|c|}
\hline $\begin{array}{c}\text { GRAD0 / } \\
\text { EDAD }\end{array}$ & $5^{\circ}$ & $6^{\circ}$ & $7^{\circ}$ & $8^{\circ}$ & $9^{\circ}$ & $10^{\circ}$ & $11^{\circ}$ \\
\hline 14 AÑOS & 2 & 8 & 6 & 9 & 6 & & \\
\hline 15 AÑOS & 1 & 2 & 6 & 6 & 10 & 4 & \\
\hline 16 AÑOS & & 1 & 1 & 4 & 2 & 9 & 4 \\
\hline 17 AÑOS & & 1 & & 1 & 2 & 6 & 13 \\
\hline 18 AÑOS & & & & & & 4 & 2 \\
\hline 19 AÑOS & & & & 1 & & & 1 \\
\hline $\begin{array}{l}20 \text { A } 21 \\
\text { A ÑN } \\
\end{array}$ & & & & & 1 & & 3 \\
\hline TOTAL & 3 & 12 & 13 & 21 & 21 & 23 & 23 \\
\hline \multicolumn{7}{|c|}{ TOTAL: } & 114 \\
\hline
\end{tabular}

Fuente: Autor

Tabla II. Grado de la muestra

\begin{tabular}{|l|c|c|c|c|c|c|c|}
\hline \multicolumn{7}{|l|}{} & TOTAL \\
\hline GRADO & $6^{\circ}$ & $7^{\circ}$ & $8^{\circ}$ & $9^{\circ}$ & $10^{\circ}$ & $11^{\circ}$ & 6 \\
\hline CANTIDAD & 10 & 10 & 15 & 15 & 15 & 23 & 88 \\
\hline
\end{tabular}

Fuente: Autor 
El diseño utilizado en la investigación correspondió a un diseño de campo, ya que los datos fueron obtenidos en forma directa con el personal involucrado en el estudio y el instrumento aplicado para la recolección de datos fue la entrevista, dada la importancia que tiene el investigador para acercarse al fenómeno en estudio y extraer de ellos información, y una vez recopilada la información se procedió a la organización de los datos en forma sistemática, para su posterior tabulación, revisión y verificación, logrando de esta forma la obtención de tablas de frecuencia que resumen la información de forma independiente de las variables involucradas en la investigación.

Desde lo teórico, se expuso en esencia la teoría humanista jerárquica de las necesidades de [11], sin embargo, también el enfoque constructivista desde el punto de vista del aprendizaje colaborativo [12], el enfoque humanista desde el punto de vista [13] aportando la historia, el concepto y el método desde el planteamiento estratégico personal y finalmente los aportes desde los proyectos de vida y planteamiento estratégico personal [14].

Figura 1. Pirámide de Maslow: Jerarquía de Necesidades

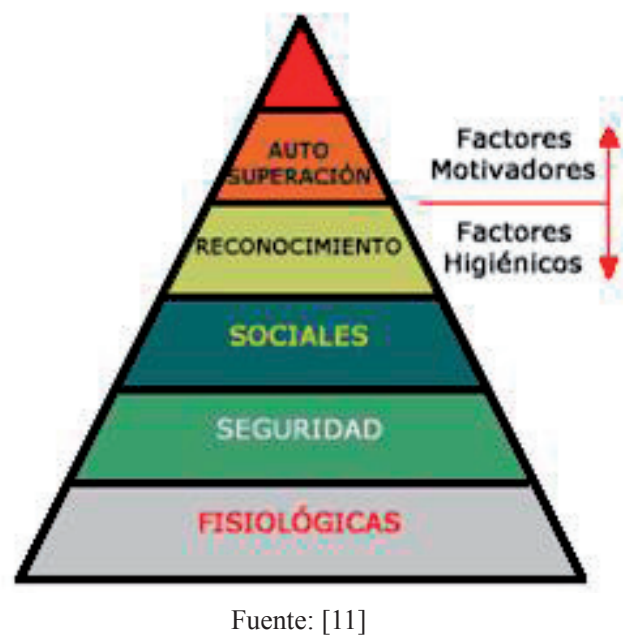

A partir de estos fundamentos teóricos se construyó el instrumento con el interés de lograr los objetivos propuestos en la investigación. A continuación, se describen los datos de identificación y los factores abordados.

- Datos generales, entendidos como datos personales, datos familiares, datos socioeconómicos y de experiencia escolar.

- Las expectativas personales

- Los factores intrínsecos que influyen en su proyecto de vida

- Los factores extrínsecos que influyen en su proyecto de vida

- Las tendencias profesionales y ocupacionales.

De donde cada punto mencionado anteriormente se presentaba en algunos casos preguntas para seleccionar y en algunos casos situaciones para jerarquizar a partir de lo establecido, en cualquiera de los casos fueron temas que nos permitieron caracterizar la población adolescente en el área metropolitana de San José de Cúcuta en relación con la construcción de su proyecto de vida.

Así mismo, de acuerdo con el objetivo general de la investigación, los resultados y el análisis de las entrevistas realizadas y tomando como referencia al enfoque humanista, se puede comprender fácilmente que, en su teoría, el ser humano está impulsado por una tendencia a la autorrealización, es decir, que dicho enfoque considera al hombre como dotado de todas las potencialidades necesarias para su completo desarrollo. Así mismo, los teóricos humanistas ven la vida como un proceso en el que luchamos por realizar nuestro potencial y por consiguiente que la personalidad es un aspecto de los esfuerzos que hacemos por desarrollar y realizar.

Ahora bien, con los adolescentes objeto de estudio, se puede corroborar ese aspecto inherente del ser humano, que busca su autorrealización, pues, desde su etapa escolar, van reconociendo la importancia de trazar metas y enmarcarlas en un plan, que, aunque no sea escrito o estructurado, si aportarán una delimitación en el desarrollo 
de sus capacidades y por ende de su ser en la búsqueda de un bienestar, en este caso, propio. La muestra con la cual trabajamos, enmarcados en el contexto escolar del Corregimiento de San Faustino, desde su condición estudiantil, se ha familiarizado con la definición y elaboración de proyectos de vida, ya que viven experiencias de formación, en las cuales van explorando sus aptitudes y potenciales al tiempo que comienzan a idealizar un futuro, del cual son conscientes que solo se construye con esfuerzo, aún desde las tareas más pequeñas que se van trazando. $\mathrm{Y}$ es a esto precisamente que denominan proyecto de vida.

En cuanto a la identificación de los factores intrínsecos y extrínsecos que influyen en el proyecto de vida de los adolescentes de la zona metropolitana, ha sido altamente formativo reconocer en la población escolar rural trabajada, una gran claridad en esos factores específicos que enmarcan el impulso que los conlleva a la elaboración de sus proyectos de vida. A pesar que los adolescentes participantes de la investigación son de corta edad y no demostraban anteriormente una línea o área de conocimiento a seguir, con la aplicación del instrumento dejaron entrever cada una de sus expectativas que resplandecen como bombillos intermitentes, enviando impulsos no sólo a su cerebro, sino también a su corazón y el resto de su ser. Cada uno de ellos demostraba las razones o motivos claves que les permitía definirse en el momento en que con propiedad hablaban de sus proyectos de vida.

De alguna u otra forma se dejaban ver como entes en función de una serie de estímulos, no sólo provenientes del medio sino también de su propio interior. No deciden por azar, todas sus metas responden a experiencias, a estereotipos de su alrededor, responden a expectativas de lo que es novedoso para ellos. Y en el más realista de los casos, responden a sus posibilidades sociales y económicas, las cuáles son enmarcadas por el núcleo familiar en el cual interactúan. Lo anterior permite reconocer que a su corta edad ya reconocen claramente y comprenden la diferencia entre realidad e ilusión, sin dejar de lado en ningún momento, aquella esperanza que puede ser mucho más fuerte que la razón. Priorizan en su necesidad de superación, necesidad de cambiar su realidad; sueñan con satisfacer sus necesidades y por encima de todo, minimizarlas, mejorando notablemente su calidad de vida y por ende el logro de un futuro asegurado.

Ante lo anterior, se establece una estrecha relación con la teoría humanística de autorrealización, de [9], quien establece una jerarquización de necesidades, las cuales, según él, el hombre va satisfaciendo en la medida en que se lo propone. Aquí se reconoce que cada individuo establece sus necesidades en grados de importancia y para contrarrestarlas, traza metas de una forma activa y pertinente. Ahora bien, la necesidad de Seguridad que [15] enuncia, la define como un estado de orden y estabilidad, reconociendo el concepto de mantener el "control de sus vidas" previniéndose de lo desconocido y de la anarquía.

Podemos inferir que, los estudiantes del Colegio Fray Manuel Álvarez son adolescentes que tienen claro que es un proyecto de vida y a partir de este concepto establecen metas anidadas en sus anhelos de tener una mejor calidad de vida, por ello, ante la finalidad de establecer la tendencia profesional $\mathrm{u}$ ocupacional de los proyectos de vida de los adolescentes, se entrevé su lucha por el alcance de sus metas, al tiempo que dejan comprobar que tienen claro que un camino seguro para lograr esa calidad de vida es la preparación intelectual y le apuntan a querer prepararse a nivel profesional a partir del reconocimiento de sus habilidades intelectuales. Son conocedores, además, que la educación superior en Colombia según
Enero-Diciembre 2016 ISSN 1794-8231 E-ISSN 2462 - 8794 PP: $112-120$

\section{7}


la ley 30 es un servicio público cultural, inherente a la finalidad social del Estado y que por tal motivo el estado puede ofrecerlo algún tipo de apoyo para la realización de estudios superiores.

En el caso de los adolescentes participantes de esta investigación, demuestran una gran inclinación por tres áreas del conocimiento, en el siguiente orden: ingeniería [16], la economía y la veterinaria. Estas áreas del conocimiento, no son ajenas a los adolescentes, pues se relacionan con las actividades económicas del sector, y a las características e implicaciones que el contexto rural fronterizo en el cual habitan, les ofrece.

Ante lo anterior y para desventaja de los adolescentes que conforman la muestra del presente proyecto, la institución Educativa en la cual se encuentran, no se ha apropiado de un programa específico para la orientación de la construcción de proyectos de vida, sino que maneja actividades de forma transversal y simultáneas, que buscan el reconocimiento de afinidades, y aptitudes que ayudarán a los adolescentes a la planeación de algunas metas para sus vidas. La planeación de dicho programa, elevaría notablemente el desarrollo integral de sus educandos, proyectando en ellos, el reconocimiento oportuno de sus potencialidades y una mejor visualización de su futuro en función de mejorar la calidad de vida de los estudiantes y de sus familias.

\section{Conclusiones}

118
A partir de la información recolectada se identifica una gran inclinación por las ingenierías como una tendencia marcada entre los estudiantes para continuar sus estudios de formación profesional, se puede deducir que esta inclinación está dada por las características propias del contexto del corregimientos pues una de las actividades principales productivas es la minería y aunque hasta el momento la vinculación laboral se encuentre únicamente como obreros, ellos demuestran interés por incluir en su proyecto de vida una profesionalización como ingenieros para ascender laboralmente en este aspecto.

Así mismo, otra de las tendencias profesionales que se encuentran marcadas en la información que brindaron los adolescentes, son las ciencias empresariales concluyendo que se deriva de otra de las actividades económicas del contexto, pues el comercio con el país vecino, Venezuela, marca el estilo de vida de algunos habitantes que a su vez muestran interés por ampliar la relación comercial binacional aprovechando su ubicación geográfica.

Finalmente, como tendencia profesional se encuentra la veterinaria, que también es derivada de la actividad agraria del contexto del corregimiento, pues, siendo zona rural existen campos que se adaptan a la reproducción ganadera y sus derivados, además vale aclarar que muchas familias lo tienen como tradición y ellos desean continuarla.

Sin embargo, se puede mencionar que no solo el contexto define la tendencia profesional en el proyecto de vida de los estudiantes [17], sino que también existen factores intrínsecos como lo son sus expectativas, su deseo de superación, sus necesidades básicas, los valores morales y religiosos, aspectos relacionados con su salud, entre otros que son determinantes en el momento en que ellos expresan al plantear su proyecto de vida.

Vemos que, para algunos adolescentes, sus familias son las que marcan de manera directa su futuro profesional, pues son quienes aportan económica y moralmente las condiciones en que podrían continuar su formación académica [18].

Es importante reconocer que los adolescentes tienen claro que tener un proyecto de vida planteado y establecido mejora la calidad de vida no sólo de su familia sino la de propia y 
que de esta manera aportan significativamente a la construcción de una sociedad más justa y en paz.

\section{Agradecimientos}

A todos y cada uno de los directivos, docentes y en especial a los adolescentes de la Institución Educativa Colegio Fray Manuel Álvarez del corregimiento de San Faustino, área rural del municipio de San José de Cúcuta, por permitir hacerles la orientación de sus proyectos de vida.

\section{Referencias}

[1] A.H. Maslow, "A theory of Human Motivation", Psychological Review, no. 50, pp.370-396, 1943.

[2] M.V.Besora, "La psicología 'humanista: historia, concepto y método". Anuario de psicología, vol. 34, pp.7-46, 1986.

[3] T.R. Vargas, Proyecto de vida y planeamiento estratégico personal, Lima, Perú. 2005

[4] F. Savater, Las preguntas de la vida. Barcelona: Mc Graw Hill, 1999, pp. 49.

[5] O.D' Ángelo, "Proyecto de vida como categoría básica de interpretación de la identidad individual y social", Revista Cubana de Psicología, vol. 17, no. 3, 2000

[6] J.N. Ramón Suárez, Formación de competencias para salir de la pobreza en modelos educativos rurales, Buenos Aires: CLACSO, 2010, pp. 132.

[7] R. Cifuentes y B. Gómez, (2012), Plan de vida [En línea]. Disponible en http:// www.redseguraclinicas.com/mujeres 1/ mujeres-gu/index.php?option $=\mathrm{com}$ content\&view $=$ article\&id=146:plande-vida\&catid=49: noticias \& Item $\mathrm{id}=169$.
[8] O. Reina, "Los proyectos futuros de la motivación: una mirada desde la psicología", Contribuciones a las Ciencias Sociales, vol. 1, no. 1, p. 1, 2010.

[9] P.J. Santos and P. Olmos, “Orientaciones para la intervención ante los factores de riesgo asociados al fracaso escolar de los jóvenes en la educación secundaria obligatoria", Revista Iberoamericana de Educación, no. 58/3, 2012.

[10] W. Verdugo, (2010, octubre, 05), Investigación descriptiva [En línea]. Disponible en http://www.slideshare. net/wenceslao/investigaciondescriptiva-5366924

[11] Wikipedia, (2012), Pirámide de Maslow [En línea]. Disponible en http://es.wikipedia.org/wiki/ Pir\%C3\%A1mide_de_Maslow

[12] J.J.C. Cardona,” Los aprendizajes colaborativos como estrategia para los procesos de construcción de conocimiento", Educación y Desarrollo Social, vol. 4, no, 2, pp.87-103, 2010.

[13] M. Villegas y I. Besora, "La psicología humanística: historia, concepto $\mathrm{y}$ método". Anuario de Psicología, vol. 1, no. 34, pp. 7-45, 1986.

[14] T.R, Vargas, Proyecto de vida y planeamiento estratégico personal, Lima, Perú, 2005. A. Maslow, Toward a psychology of being. Nueva York: J. Wiley and Sons, 1968, pp. 270.

[16] J. Martínez, M. Vergel y L. Zafra, Comportamiento Juvenil $y$ competencias prosociales, Bogotá: Ibáñez, 2015. 
Eco.Mat

No. 1

Enero-Diciembre 2016 ISSN 1794-8231
Proyecto de vida de los adolescentes del área metropolitana de Cúcuta (zona rural escolar corregimiento de

[17] C. Acevedo, M, Vergel y E, Flórez, Teoría de contacto aplicada al mecanismo Leva-palpador Cilíndrico, Bogotá: Editorial Universidad Nacional de Colombia, 2012, p.58.

[18] M. Cárdenas, M. Vergel y O. Rincón, "Reliquidación de Matrícula. Caracterización del beneficiario en la Universidad Francisco de Paula Santander", Revista Ecomatemático, vol. 4, no. 1, pp. 80-94, 2013. 